\title{
La révision dans la rédaction conversationnelle : opérations mobilisées par deux étudiants de deuxième année de licence de français
}

\section{Résumé:}

Ce travail de recherche porte sur les opérations de révision utilisées par deux étudiants de deuxième année de licence français, à l'université de Jijel, au cours de la rédaction conversationnelle, d'un texte argumentatif. Il ne s'agit nullement pas de répertorier les modifications apportées au brouillon mais plutôt d'étudier la mobilisation, en temps réel, des différentes opérations qui sont sous-jacentes à ces modifications.

Notre corpus, constitué d'une rédaction conversationnelle, fera l'objet d'une double analyse, qualitative et quantitative. L'analyse qualitative nous permettra de définir les opérations de révision, de préciser le niveau textuel et la localisation des différentes modifications dans toutes les phases de la rédaction. Elle nous permettra également de voir la construction progressive, dans le temps et dans l'espace, de chaque segment du texte. L'analyse quantitative quant à elle, nous permettra de voir la fréquence d'utilisation des opérations de révision et de préciser quelles sont les plus ou moins récurrentes.

Mots clefs : révision, stratégie, opération, processus, rédaction conversationnelle (rédaction collaborative).

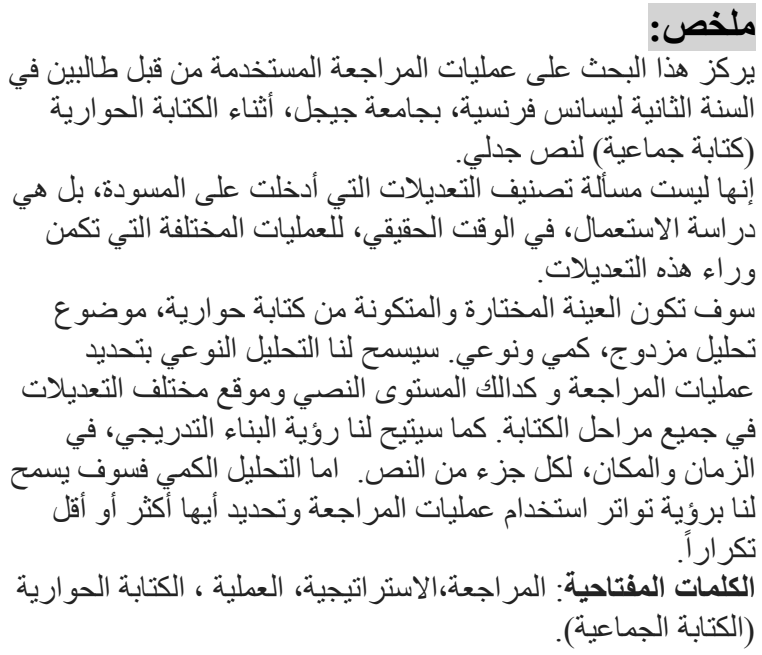

\section{Wided ASSILA}

Département des lettres et langue française Université de Jijel

\author{
Introduction: \\ «Révision » est un terme \\ ambigu qui jusqu'à ce jour, n'a \\ pas pu obtenir une définition \\ univoque. En effet, depuis plus \\ d'une décennie, de nombreuses \\ recherches se sont attachées à \\ décrire et à analyser l'activité de \\ révision.
}


Elle est conçue par certains comme le prolongement du travail d'écriture, par d'autres comme un ensemble d'opérations allant de la simple relecture à la reformulation complète d'un texte. (J.David, 1994:3) On note de ce fait, l'existence de deux dénominations différentes attribuées à cette activité : révision et réécriture. La première est liée à la génétique textuelle alors que la seconde relève de la psychologie cognitive.

$\mathrm{Si}$ les chercheurs ne sont pas parvenus à s'accorder sur une définition consensuelle de la révision, ils s'accordent sur son importance et sa nécessité dans la rédaction d'un texte. (Sommers 82 cité dans Bisaillon, $91: 11$ ) Car la réécriture n'est pas seulement une activité de réparation qui diminuerait avec l'augmentation de l'efficacité rédactionnelle, elle est constitutive de l'expertise.

Dans cet article, nous avons essayé d'étudier l'activité de révision et ce à travers l'analyse des différentes opérations employées au cours de cette activité. Notre étude s'inscrit dans le cadre de la psychologie cognitive qui considère la rédaction comme un ensemble de trois processus récursifs : planification, mise en texte et révision. Par ailleurs, cette étude s'appuie sur une méthode d'observation qui est celle de la rédaction conversationnelle, car notre recherche dépasse le cadre des seuls produits (achevés ou brouillons) ; nous voulons voir la mobilisation en temps réel des différents processus rédactionnels, et en particulier, observer ce que font les étudiants quand ils révisent leurs textes et la manière dont ils gèrent ce processus. À notre sens, seule la rédaction conversationnelle nous permet d'atteindre un tel objectif, grâce à l'interaction qui oblige les partenaires à expliciter chacune de leurs activités.

Cette recherche tente d'apporter des éléments de réponse à un certain nombre de questions : -La procédure de révision a-t-elle un impact sur la qualité de révision? -Que font les étudiants quand-ils révisent leurs textes ? Sont-ils capables de lui apporter des modifications de fond ou celles-ci concernent uniquement la surface? -Les étudiants mobilisent-ils toutes les opérations requises par l'activité de révision? -Si oui, quel est le temps et quelle est l'importance accordés à chacune d'entre elles ?

Trois hypothèses fondamentales sont à la base de notre recherche : -L'attention des étudiants est centrée sur la révision des éléments de surface que sur les éléments de fond. -Les étudiants utilisent des opérations de révision mais qui diffèrent en nature et en fréquence. -Les étudiants sont généralement incapables de détecter les erreurs au niveau du texte et même s'ils arrivent à le faire, ils sont incapables de trouver les corrections adéquates. 


\section{1- Quelques définitions :}

1.1- La révision selon la psychologie cognitive :

Michel Fayol (2002:54) donne une définition très simple de la révision. Pour lui elle consiste à relire le texte produit ou encours de production et lui apporter les modifications nécessaires. Bien que cette définition paraisse simple, plusieurs modèles de la révision ont été proposés dans le cadre de la psychologie cognitive. Nous citons : les modèles de Hayes et Flower (1980), Breiter et Scardamaia (1983), Hayes et Al (1986), Hayes et Al (1996), Butterfield et al (1996). Dans cette recherche, nous avons choisi de présenter le modèle de révision de Hayes de 1987. Notre choix de ce modèle se justifie par le fait que ce dernier insiste sur les options stratégiques qui se présentent au rédacteur

\subsection{1- Le modèle de Hayes, Flower, Schriver, Stratman et Carey (1986) :}

Dans ce modèle, la révision commence par une lecture critique du texte écrit pour détecter les anomalies qu'il peut contenir. Si un problème est découvert, le scripteur l'évalue et choisit ensuite l'une des stratégies de révision selon l'importance du problème telle que le rédacteur l'a évaluée après une lecture attentive de son texte. " Il peut alors ignorer le problème jugé trop difficile à traiter ou peu important » ( Piolat et Roussey, 1994: 50), « il peut différer la recherche d'une solution, ou encore chercher plus d'informations en mémoire ou dans le texte pour le comprendre et mieux le définir, de réécrire le texte ou un fragment de celui-ci en préservant l'idée de base, ou encore de réviser le texte afin de préserver au maximum le texte déjà produit ». ( Alamargot et Chankoy in Fayol, 2002 : 55) Une fois que le rédacteur entame la réécriture, il effectue plusieurs modifications qui différent selon : «a- le type d'opérations réalisées pour y parvenir (addition, suppression ou substitution de mots, de groupes de mots ou de phrases, modification d'une partie du texte, etc.), b- le niveau textuelle auquel ces modifications se situent ( en surface ou en profondeur), c- leur localisation dans le texte (début, milieu, fin) et dans la phase de composition ( écriture d'un plan, d'un brouillon, de la version finale, lors d'une étape spécifiquement révisionnelle, etc.) (Alamargot et Chankoy in Fayol, $2002: 55$ )

Le modèle de Hayes et al.(1986) est un modèle original. Son originalité est issue du fait qu'il a présenté la révision comme un processus autonome, et l'a étudiée indépendamment de ses interactions avec les autres processus de la révision. De plus et grâce à «son caractère procédurale et à la finesse de définition », il a inspiré d'autres chercheurs et leur a permis d'élaborer d'autres modèles comme celui de Butterfield, Hacker et Albertson (1996). (Alamargot et Chankoy in Fayol, dir., 2002 : 55) 


\section{2- Les opérations de révision :}

Pour Hayes (1986), ainsi que pour plusieurs autres chercheurs dont Faigley et Witte (1981), Monhan (1984), Grésillon et Lebrave (1983), Fabre (1990), si le rédacteur s'engage dans la réécriture de certains aspects de son texte, plusieurs opérations lui sont alors offertes: l'ajout, la suppression, le remplacement et le déplacement. Cette classification a été faite en fonction de trois dimensions : la nature des modifications, leur site et « la profondeur » relative du changement introduit. ( Fayol, 1997: 132; A. Moulin et al. in Marie Madelaine de Gaulmine et Robert Bouchard, 2001 :251)

\subsection{1- L'ajout :}

Pour Claudine Fabre (2002: 84), l'ajout se définit comme l'insertion d'un élément $X$ dans un texte qui existe déjà. . Ainsi, « la séquence $A B$ du premier état devient l'une des séquences $\mathrm{XAB}, \mathrm{AXB}$ ou $\mathrm{ABX}$ dans l'un des états suivants $»$. Cette insertion a une position bien définie au niveau de la page écrite. Elle peut se situer « à l'entour du texte, entre les lignes, voire entre les mots, dans les espaces laissés blancs par le texte préalablement». (Claire Doquet, Christophe Leblay, 2014 :7)

\subsection{2- La suppression :}

Si l'ajout consiste à insérer dans le texte un élément nouveau, la suppression quant à elle consiste à effacer un élément qui existe déjà dans le texte, sans qu'il soit remplacé par un autre élément. Ainsi, « la séquence AXB devient la séquence $\mathrm{AB}$ (suppression simple de $\mathrm{X}$ ) (Fabre, 2002 : 109).

\subsection{3- Le remplacement :}

Claudine FABRE (1990) définit le remplacement comme " l'opération par laquelle un élément langagier est supprimé, tandis que un autre élément est ajouté pour se substituer au premier, de sorte que l'un est l'autre fonctionnent comme équivalent». (E.Chandelier, 2004: 12) cette définition a été représentée de la manière suivante : " une séquence initiale $\mathrm{AXB}$ devient la séquence modifiée $\mathrm{AYB}$, dans laquelle $\mathrm{A}$ et $\mathrm{B}$ désignent le contexte langagier immédiat, et $\mathrm{X}$ et $\mathrm{Y}$ les éléments entrant dans l'opération de remplacement » (Fabre, 2002 : 59).

\subsection{4- Le déplacement :}

Selon Fabre (2002: 133), le déplacement est une opération «marquée entre deux états d'un écrit lorsqu'une séquence $\mathrm{AXB}$ apparaissant dans l'une des versions se trouve remplacée dans l'autre par la séquence $\mathrm{XAB}$ ou $\mathrm{ABX}$ ». Il s'agit en d'autres termes de relever un élément du texte pour le placer dans un autre emplacement jugé plus adéquat. (Aurélie Mounier, 2016 : 16)

\section{3- La rédaction conversationnelle :}

La rédaction conversationnelle peut être définie comme une situation d'écriture mettant en évidence les différents processus. (M-M de Gaulmyn et al., 2001 : 9). 


\section{La révision dans la rédaction conversationnelle : opérations mobilisées par deux étudiants de deuxième année de licence de français.}

En termes plus simples, la rédaction conversationnelle n'est qu'une activité d'écriture en collaboration. Elle permet d'observer la situation où se conjointent l'oral et l'écrit, plus précisément la situation où l'oral sert à produire l'écrit. Cette dernière «offre un point de vue privilégié sur le processus de production écrite et sur la mise en œuvre de la compétence plurielle, écrite et orale, du locuteur lettré ». (M-M de Gaulmyn et al., 2001 : 9)

La rédaction conversationnelle est, dès 1991, au centre d'intérêt de l'équipe Gric 2 «interaction, acquisition, apprentissage» des langues. Cette équipe appartient à un laboratoire de science du langage de l'université de Lyon II. Elle a une orientation didactique et s'intéresse l'étude des interactions directement à l'enseignement et l'apprentissage des langues. L'intérêt pour la rédaction conversationnelle, nous précise de Gaulmyn revient au fait que les situations oralo- graphiques sont méconnues. En effet, l'analyse des textes écrits a toujours été disjointe de celle du langage parlé et également a toujours porté sur l'étude des produits finis de l'écriture et non sur les processus de leur production. Alors que les situations de rédactions conversationnelles sont des situations ordinaires, comme par exemple les activités à caractère professionnel ( $2001: 31-34)$ surtout avec l'insertion des systèmes coopératifs associés à des réseaux hauts débits dans les différentes organisations. A.Moulin et al. (2001: 248) signalent à ce sujet que l'utilisation des collecticiels comme les visioconférences ou les éditeurs partagés favorisent la réalisation de projets au niveau international. Ils expliquent que «plusieurs groupes géographiquement distants peuvent ainsi réaliser des travaux de téléécriture collaborative sans se soucier ni de l'espace ni du temps (....) Ce type de médiatisation technologique plusieurs formes : elle peut être visuelle ou textuelle et elle permet une communication en temps réel dite synchrone (visioconférence avec partage d'application) ou une communication différée dite asynchrone ( courrier électronique, forum ....)». (ibid)

\section{2- Méthodologie :}

L'objectif de cette recherche est de mettre en relief les différentes opérations sous-jacentes aux modifications d'un texte produit ou en cours de production. Pour ce faire nous avons effectué une double analyse, qualitative et quantitative. La première, essentiellement descriptive, nous permet d'étudier la mobilisation en temps réel des différentes opérations conduisant à des modifications du texte et de préciser la localisation et le niveau textuel de ces modifications. L'analyse quantitative quant à elle, est de nature statistique. Elle nous permet de connaitre la fréquence de l'utilisation de chacune des opérations de révision.

L'analyse qualitative s'est déroulée ainsi : nous avons d'abord segmenté notre corpus en fonction des différentes étapes de la rédaction: la planification, 
l'élaboration d'un brouillon, la relecture du brouillon, l'inscription du texte au propre et la révision finale. Dans chacune de ces phases, nous avons sélectionné les extraits du corpus contenant des révisions. De ces extraits, nous avons dégagé toutes les opérations effectuées tout en décrivant la manière dont s'effectuent les transformations que subissent les différentes unités du texte et à préciser et l'emplacement et la localisation de chacune de ces modifications. Enfin, nous avons essayé de représenter la formulation progressive de chaque phrase du texte.

Les résultats de l'analyse qualitative sont complétés par une analyse quantitative dont l'objectif est précisément d'étudier la fréquence du recours aux différentes opérations de révision. L'analyse quantitative s'est déroulée de la manière suivante : nous avons d'abord compté le nombre des occurrences de chacune des opérations de révision, d'abord au niveau de l'ensemble de la rédaction et en suite au niveau de chacune de ses étapes, à savoir, la phase de planification, de la composition du brouillon, de la relecture du brouillon, de la transcription de la version finale et enfin de la relecture finale du texte produit. Les résultats obtenus ont fait par la suite, l'objet d'une comparaison afin de préciser quelles sont 1 opérations de révision les plus ou moins récurrentes.

\section{3- Le corpus :}

Notre corpus est constitué d'une rédaction conversationnelle d'un texte argumentatif, réalisée par deux étudiants, une fille et un garçon, de deuxième année de licence de français à l'université Tassoust de Jijel. Ils devaient répondre à la consigne suivante: «Faut-il battre les enfants pour les éduquer ? Qu'en pensez-vous?». Ces étudiants devaient disposer de 90 minutes pour rédiger ensemble leur texte, mise au propre comprise. L'enregistrement total que nous avons recueilli compte une heure et demie et la transcription complète occupe 23 pages. Les tours de paroles sont en nombre de 474 .

\section{1- Les conventions de transcription :}

Pour transcrire notre corpus nous avons adopté les conventions de transcription utilisées par Annie Chalivet en 1995-1996 pour transcrire une rédaction conversationnelle. A noter nous avons apporté quelques petits changements à ces conventions. Nous avons également inséré d'autres signes pour symboliser les passages en langue arabe ainsi que leur traduction.

\begin{tabular}{|c|c|}
\hline $\mathrm{F}$ & l'étudiante \\
\hline G & l'étudiant \\
\hline$=$ & élision \\
\hline - & troncation de mot \\
\hline & allongement \\
\hline Souligné & chevauchement \\
\hline$\underset{/}{\text { E s p a c é }}$ & $\begin{array}{l}\text { articulation lente } \\
\text { interruption }\end{array}$ \\
\hline
\end{tabular}


La révision dans la rédaction conversationnelle : opérations mobilisées par deux étudiants de deuxième année de licence de français.

$\begin{array}{ll}0 & \text { pause courte } \\ 00 & \text { pause mi-longue } \\ 000 & \text { pause longue } \\ \text { (silence) } & \text { pause très longue } \\ (\text { xxxxx) } & \text { passage inaudible ou incompréhensible } \\ (\quad) & \text { activité non verbale } \\ & \text { intonation montante } \\ & \text { intonation descendante } \\ \text { Maj } & \text { passage en langue arabe } \\ \text { ( Maj) } & \text { traduction du passage en langue arabe }\end{array}$

Graphèmes correspondant à certains phonèmes de la langue arabe :

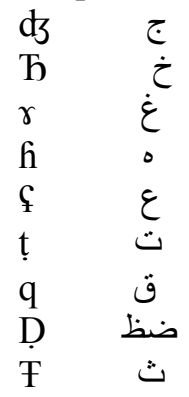

Les opérations de révisions sont désignées par les symboles suivants :

Suppression : ABC

Remplacement : $A B C(D E F)$

Ajout : + (ABC)

Déplacement: $(\mathrm{ABC}) \quad \longrightarrow(\mathrm{ABC})$

4- Le public :

Le public que nous avons choisi pour mener notre recherche consiste en deux étudiants de deuxième année de français, une fille (F) et un garçon (G) âgés de 20 ans. Ces derniers sont tous les deux algériens et poursuivent leurs études à l'université Tassoust de Jijel.

\section{5- Segmentation du corpus}

- Du T1 à T18: Le plan général du texte

Les co-scripteurs commencent par établir verbalement le plan du texte à produire. Ils décident alors de commencer par une introduction qui tourne autour du thème des enfants. Cette introduction sera suivie d'une problématique posée sous forme d'une question. Ensuite sera présenté le développement dont le rôle est de ramener des éléments de réponse à la problématique et ce par le biais de différents arguments. En fin le texte à produire se termine par une conclusion. Une fois le 
plan établi, les co-scripteurs se mettent d'accord sur la manière de gérer la tâche, et ce au niveau du tour de parole 19.

- T19: G : Donc chacun va donner une idée

Il importe de signaler que le plan établi par nos collaborateurs consiste en réalité en une définition des différentes parties du texte à produire. A l'exception de l'introduction, le contenu du développement et de la conclusion n'a pas été défini au cours des discussions. En conséquence et à notre sens, ceci va leur poser des problèmes lors de la rédaction et les obliger à investir beaucoup de temps pour la recherche des idées.

- Du T20 à T97 : Rédaction de l'introduction au brouillon.

- Du T98 à T394 : Rédaction du corps du texte.

- De T114 jusqu'à T371 : Rédaction des arguments qui sont en nombre de trois.

- De T372 à T394 : Rédaction de la conclusion.

- De T395 à T473: Relecture de texte écrit sur le brouillon, modification de celui-ci et écriture de la version finale.

- Le tour de parole T474 indique la fin de la rédaction par la formule de soulagement de $\mathrm{F}$ " ah baba » qui montre combien a été difficile la production du texte.

\section{6- Analyse des opérations de révision :}

\section{1- Analyse qualitative :}

Nous présentons dans ce qui suit un exemple de révision de la troisième phrase de l'introduction.

\section{Extrait 7 : Révision 7 :}

$72 \mathrm{~F}: \mathrm{mhm}, 00$ les enfants nés dans nés dans une société dans une où la violence YA RABI (OH MON DIEU)je ne trouve pas la formule 0 la violence 0 règle tout 0 résolue tout 0 oh il me faut un verbe un verbe $0 \mathrm{j}$ 'allais dire nés dans une société pourrie pourrie ( rire) mais mais les enfants nés dans une société où la violence 00

73 G: est donc le moyen qu'on a 0 qu'on a toujours recours 0 pour régler les problèmes

$74 \mathrm{~F}$ : non, 0 il me faut juste un verbe ici $0 \mathrm{pa}=\mathrm{c}=$ que la phrase $\mathrm{hEDI} E Y$ MLIHA (ELLE EST BONNE CELLE-LA)

$75 \mathrm{G}: \mathrm{mhm}$,

76 F : ( relie) les enfants nés dans une société où la violence est $\mathrm{p}: 000$ ah ça m'énerve $000 \mathrm{mhm}$ euh 00 résoudre 0 le verbe 00 comment on conjugue résoudre "

$72 \mathrm{G}$ : résolu 0 résolution 

deux étudiants de deuxième année de licence de français.

73 F : la violence résolue 00 MAৎANDAKCH ( TU N'AS PAS) dictionnaire'

74 G : non,

75 F : oh la la ( silence) ah 00 on trouvera on trouvera ce verbe

$\mathrm{F}$ vient de te terminer l'écriture des deux premières phrases de l'introduction. $\mathrm{G}$ lui signale de poser la question directement après ces deux phrases. Mais $F$ lance une nouvelle idée pour l'introduction, en recourant à la langue arabe puisqu'elle éprouvait des difficultés à le faire en français. Elle formule au (T 72) une partie d'une phrase complexe « Les enfants nés dans une société où la violence », mais trouve des difficultés à la compléter.

Le problème rencontré à ce niveau concerne le choix du verbe adéquat pour relier ce qui vient d'être énoncé avec ce qui va être formulé. F propose le verbe conjugué au présent de l'indicatif «règle», le remplace directement par « résolue » mais juge que ces deux propositions ne sont pas les bonnes. G essaye de résoudre ce problème en proposant une expansion à la phrase de $\mathrm{F}$ ( $73 \mathrm{G}$ : est donc le moyen qu'on a 0 qu'on a toujours recours 0 pour régler les problèmes) mais ça proposition a été tout de suite rejetée par $\mathrm{F}$ qui insiste sur ce verbe qui lui manque parce qu'elle juge que sa phrase est bonne: (74 F : non, 0 il me faut juste un verbe ici $0 \mathrm{pa}=\mathrm{c}=$ que la phrase fEDI EY MLIHA ( C'EST BIEN). F relit sa phrase pour essayer de trouver ce verbe noyau. Elle propose « est » est s'arrête un moment. Ce silence dans cette situation est une évaluation négative du choix du verbe "être » et aussi une réflexion pour résoudre ce problème. L'expression de $\mathrm{F}$ « ah ça m'énerve » montre bien le cout de la révision et aussi celui de la rédaction. (76 F : ( relie) les enfants nés dans une société où la violence est $\mathrm{p}: 000$ ah ça m'énerve $000 \mathrm{mhm}$ euh 00 résoudre 0 le verbe 00 comment on conjugue résoudre "). F se montre tenace, elle revient à sa deuxième proposition, le verbe "résoudre", mais ne sait malheureusement pas comment le conjuguer. Elle demande de l'aide à son partenaire qui lui aussi ignorait la conjugaison du verbe "résoudre». En conséquence, F veut recourir à un dictionnaire mais tous les deux n'en avaient pas. Après un moment de réflexion, $\mathrm{F}$ adopte en fin une stratégie après plusieurs tentatives vaines: elle décide de reporter la solution du problème et revenir en conséquence à la première formulation «Les enfants nés dans une société où la violence règle tout ».

\section{Représentation des différentes opérations effectuées :}

Les enfants nés dans une société où la violence règle tout(résolue tout >est donc le moyen qu'on a on a toujours recours pour régler les problèmes) est (résoudre)(règle tout) 
On peut dire que cette troisième phrase est passée par cinq versions sans qu'elle soit en fin mise en forme.

Opérations effectuées :

\begin{tabular}{|c|c|c|}
\hline Opération & Niveau textuel & localisation \\
\hline 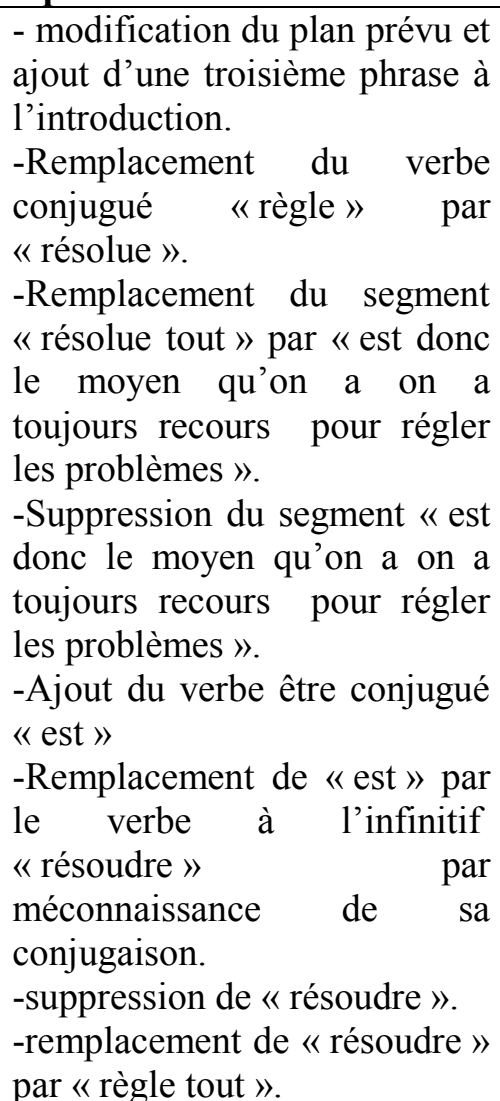 & surface & $\begin{array}{l}\text { dans le début du } \\
\text { texte, dans la phase } \\
\text { d'écriture } \\
\text { brouillon. (après la } \\
\text { fin de la formulation } \\
\text { orale de la } 3^{\text {ème }} \\
\text { phrase } \\
\text { l'introduction. }\end{array}$ \\
\hline
\end{tabular}


La révision dans la rédaction conversationnelle : opérations mobilisées par deux étudiants de deuxième année de licence de français.

Résultats de l'analyse qualitative :

\begin{tabular}{|c|c|c|c|}
\hline$n^{\circ}$ révision & Opération & Localisation & $\begin{array}{l}\text { Niveau } \\
\text { textuel }\end{array}$ \\
\hline 1 & Remplacement & $\begin{array}{l}\text { Ecriture du brouillon: } \\
\text { début du texte, dans la } \\
\text { phase de la formulation } \\
\text { orale de l'introduction }\end{array}$ & profondeur \\
\hline 2 & Ajout & $\begin{array}{l}\text { Ecriture du brouillon: } \\
\text { début du texte, dans la } \\
\text { phase de la formulation } \\
\text { orale de l'introduction }\end{array}$ & surface \\
\hline 3 & $\begin{array}{l}\text { Ajout } \\
\text { Ajout } \\
\text { Suppression } \\
\text { Remplacement }\end{array}$ & $\begin{array}{l}\text { dans le début du texte, } \\
\text { dans la phase d'écriture } \\
\text { du brouillon. } \\
\text { (L'écriture de la 1ère } \\
\text { phrase de l'introduction } \\
\text { sur le brouillon) }\end{array}$ & surface \\
\hline 4 & $\begin{array}{l}\text { Quatre } \\
\text { remplacements } \\
\text { Ajout }\end{array}$ & $\begin{array}{l}\text { dans le début du texte, } \\
\text { dans la phase d'écriture } \\
\text { du } \\
\text { (formulation orale de la } \\
2^{\text {ere phrase de de }} \\
\text { l'introduction) }\end{array}$ & surface \\
\hline 7 & $\begin{array}{l}\text { Quatre } \\
\text { remplacements } \\
\text { Deux ajouts } \\
\text { Deux suppressions }\end{array}$ & $\begin{array}{l}\text { dans le début du texte, } \\
\text { dans la phase d'écriture } \\
\text { du brouillon. (après la } \\
\text { fin de la formulation } \\
\text { orale de la } 3^{\text {ème }} \text { phrase de } \\
\text { l'introduction }\end{array}$ & surface \\
\hline 8 & $\begin{array}{l}\text { Quatre } \\
\text { remplacements } \\
\text { Quatre ajouts }\end{array}$ & $\begin{array}{lll}\text { dans le début du texte, } \\
\text { dans la phase } & \text { de } \\
\text { composition } & & \text { du } \\
\text { brouillon, lors } & \text { de } \\
\text { rédaction de } & \text { la } \\
\text { problématique. } & \\
\end{array}$ & surface \\
\hline
\end{tabular}




\begin{tabular}{|c|c|c|c|}
\hline 9 & $\begin{array}{l}\text { Quatre } \\
\text { remplacements }\end{array}$ & $\begin{array}{l}\text { dans le milieu du texte. } \\
\text { Dans la phase de } \\
\text { l'écriture du brouillon. } \\
\text { (lors de la formulation } \\
\text { orale et de l'inscription } \\
\text { de première phrase du } \\
\text { premier argument). }\end{array}$ & surface \\
\hline 10 & $\begin{array}{l}\text { Quatre } \\
\text { remplacements } \\
\text { Une suppression }\end{array}$ & $\begin{array}{l}\text { dans le milieu du texte. } \\
\text { Dans la phase de } \\
\text { l'écriture du brouillon, } \\
\text { lors de la formulation } \\
\text { orale de la } 2^{\text {ème }} \text { phrase du } \\
1^{\text {er argument }}\end{array}$ & Surface \\
\hline 11 & Suppression & $\begin{array}{llr}\text { dans la phase } & \text { de } \\
\text { composition } & & \text { du } \\
\text { brouillon, lors } & \text { de } \\
\text { l'inscription de la } & 3^{\text {eme }} \\
\text { phrase. Au milieu } & \text { du } \\
\text { texte. } & & \\
\end{array}$ & Surface \\
\hline 12 & $\begin{array}{l}\text { Sept remplacements } \\
\text { Un ajout }\end{array}$ & $\begin{array}{l}\text { au milieu du texte. Au } \\
\text { cours de la rédaction de } \\
\text { la quatrième phrase dans } \\
\text { l'étape de la composition } \\
\text { du brouillon. }\end{array}$ & Surface \\
\hline 13 & $\begin{array}{l}\text { Suppression } \\
\text { Déplacement } \\
\text { Remplacement }\end{array}$ & $\begin{array}{l}\text { au milieu du texte, lors } \\
\text { de la rédaction de la } \\
\text { cinquième et de la } \\
\text { sixième phrases à l'étape } \\
\text { de la composition du } \\
\text { brouillon. }\end{array}$ & surface \\
\hline $14,15,16$ & $\begin{array}{l}\text { Suppression } \\
\text { Treize } \\
\text { remplacements } \\
\text { Un ajout }\end{array}$ & $\begin{array}{l}\text { au milieu du texte, lors } \\
\text { de la rédaction du } \\
\text { deuxième argument à } \\
\text { l'étape de la composition } \\
\text { du brouillon. }\end{array}$ & Surface \\
\hline $\begin{array}{l}17, \\
18,19,20,21,22, \\
23\end{array}$ & $\begin{array}{l}\text { Quinze } \\
\text { remplacements } \\
\text { Quatre suppressions } \\
\text { Deux ajouts }\end{array}$ & $\begin{array}{l}\text { au milieu du texte, lors } \\
\text { de la rédaction du } \\
\text { troisième argument à } \\
\text { l'étape de la composition } \\
\text { du brouillon. }\end{array}$ & Surface \\
\hline
\end{tabular}


La révision dans la rédaction conversationnelle : opérations mobilisées par deux étudiants de deuxième année de licence de français.

\begin{tabular}{|c|c|c|c|}
\hline $24,25,26$ & $\begin{array}{l}\text { Six remplacements } \\
\text { Trois ajouts } \\
\text { Deux suppressions }\end{array}$ & $\begin{array}{l}\text { A la fin du texte, lors de } \\
\text { la rédaction de la } \\
\text { conclusion, à l'étape de } \\
\text { la composition du } \\
\text { brouillon. }\end{array}$ & Surface \\
\hline 27 & $\begin{array}{l}\text { Deux ajouts } \\
\text { Deux } \\
\text { remplacements }\end{array}$ & $\begin{array}{l}\text { A l'étape de la relecture } \\
\text { du brouillon. }\end{array}$ & Surface \\
\hline $\begin{array}{l}28,29,30,31, \\
32,33\end{array}$ & $\begin{array}{l}\text { Trois ajouts } \\
\text { Quatre } \\
\text { remplacements } \\
\text { Une suppression }\end{array}$ & $\begin{array}{l}\text { A l'étape de l'inscription } \\
\text { au propre. }\end{array}$ & Surface \\
\hline 34 & aucune & Relecture finale du texte. & \\
\hline
\end{tabular}

\section{2- Analyse quantitative :}

D'après les résultats obtenus, nous avons constaté que le texte produit a subi des révisions en nombre de 34, réparties d'une façon non équitable sur les différentes phases de la rédaction : la rédaction du brouillon a connu le plus grand nombre 26, suivi de l'écriture de la version finale avec un nombre de six. Viennent après la relecture du brouillon et la relecture finale du texte produit avec le même nombre de un, et en dernier, la phase de la planification qui n'a connu aucune révision. Ces résultats nous permettent de dire que la révision la plus importante s'effectue au moment même de la composition du texte au niveau du brouillon puisque l'écart entre la fréquence de la révision au niveau du brouillon et celle au niveau des autres phases de la rédaction est important. De plus et en ce qui concerne les différentes opérations mobilisées dans les révisions, nous avons noté la prédominance du remplacement avec un pourcentage de $64,50 \%$. A l'inverse, le déplacement, la suppression ont connu de très faibles pourcentages qui sont respectivement présentés: $1,86 \%$, $13,08 \%$. L'ajout a, lui aussi, marqué un faible pourcentage de $20,56 \%$.

De ce fait, la révision se présente comme une activité de réajustement qui accompagne pas à pas la formulation des différentes unités du texte. En ce sens que chaque unité qu'elle soit mot, groupe de mots voire phrase est objet de multiples modifications avant d'être validée. D'un autre coté, le nombre réduit des révisions au moment de la relecture finale du texte revient, à notre sens, au fait que les étudiants ne sont pas en mesure de ce distancier par rapport à leurs textes puisqu'ils lisent en fonction de ce qu'ils connaissent déjà de leurs textes. En conséquence, ils ne parviennent pas à détecter les erreurs commises. L'exemple le plus pertinent que nous pouvons citer et celui du non respect du modèle du texte argumentatif à produire. Mais le plus grand nombre 
des modifications effectuées sont des modifications de surface. En effet, les résultats auxquels nous avons aboutit montrent que 104 modifications soit 99, $\%$ touchent à des aspects formels du texte par opposition à trois modifications seulement soit $0,96 \%$ qui sont relatives au niveau profond du texte. Nous pouvons dire, à ce propos, que les étudiants quand ils révisent leurs textes, leur plus grand soucis c'est de faire correspondre leurs textes aux normes du français, plus particulièrement l'orthographe sans prendre en compte la vérification de la cohérence du texte par exemple, de son adéquation au texte demandé. En somme, réviser le texte signifie pour nos étudiants corriger les fautes d'orthographe.

\section{Conclusion:}

$\mathrm{Au}$ terme de cette recherche, nous pouvons dire que les différentes opérations de révision ont été employées par nos rédacteurs, du début jusqu'à la fin de la rédaction. Ceci dit que le processus de révision n'intervient pas au terme de la production du texte ou d'une partie du texte mais recouvre complètement le processus de mise en mot. Dans le modèle de Hayes (1987), la révision a été définie comme intervenant après une lecture critique du texte et la détection d'un problème donné. Or dans notre étude, nous avons remarqué que pratiquement, chaque phrase ou segment de phrase se confectionne par tâtonnement. Ils subissent, avant d'être validés, plusieurs modifications qui ne sont que des opérations de révisions. Ce processus ressemble en quelque sorte au jeu d'enfants " légos ». Dans ce jeu, l'enfant essaye de confectionner un objet ou une forme particulière par le biais de différentes pièces carrées ou rectangulaires, de différentes dimensions. Et pour atteindre son objectif, l'enfant commence d'abord par une pièce de base qui détermine le choix de la pièce suivante, et la deuxième pièce détermine le choix de la troisième et ainsi de suite. Et avant que l'une des pièces soit jugée comme convenable, l'enfant essaye de placer différentes pièces. Si l'une ne convient pas à la forme projetée, elle est tout de suite remplacée par une deuxième, une troisième ou une quatrième jusqu'à ce qu'il juge que l'une d'entre elle est celle qui convient le mieux à l'image de l'objet à construire. Mais si le joueur ne parvient pas monter son objet il peut y renoncer et décider de construire une nouvelle forme. De ce fait, chaque partie du texte, qu'elle soit longue ou courte est instantanément contrôlée et par suite modifiée jusqu'à ce qu'elle s'avère en harmonie avec ce qui vient d'être énoncé et répondant aux contraintes de la rédaction. Cela ne se fait pas d'une façon linéaire. La formulation et la reformulation d'une phrase par exemple, ne se développe pas dans un seul sens. Les rédacteurs peuvent par exemple valider une forme de phrase à l'étape orale mais quand ils l'écrivent, ils lui donnent une autre forme. Ils peuvent également procéder à la modification de la fin d'une phrase, produire ensuite une phrase suivante et retourner au début de la phrase précédente pour le 


\section{La révision dans la rédaction conversationnelle : opérations mobilisées par}

deux étudiants de deuxième année de licence de français.

modifier. Il est à noter que le fait de réécrire un même fragment en deux temps différents nous a été un problème à surmonter, à un certain moment de l'analyse, celui de la représentation chronologique des différentes opérations de révision. Un autre résultat auquel nous avons abouti est que la révision n'est pas toujours consécutive d'une amélioration du texte. Les corédacteurs peuvent lui apporter des corrections erronées. Ils peuvent même ne pas se rendre compte des anomalies au niveau de leur texte. A titre d'exemple, nos corédacteurs ont terminé la rédaction du texte, l'ont relu, l'on jugé comme «bon » selon leur expression alors que leur rédaction ne répond pas au modèle $\mathrm{du}$ texte argumentatif à produire. Il fallait produire un texte contenant deux thèses antithétiques, appuyée chacune par trois arguments. Le texte produit par $F$ et $G$ s'articule autour d'une seule thèse et de trois arguments.

Un autre résultat de cette recherche concerne le retour sur le texte en cours de production ou bien encore la relecture de ce dernier. Celle-ci est considérée dans la littérature de la révision comme une lecture critique du texte qui permet la détection et le traitement des anomalies au niveau du texte. Notre étude nous a montré que certaines de ces relectures sont seulement des étapes pour pouvoir enchainer ce qui vient d'être rédigé avec ce qui va être formulé.

Et pour les évaluations du texte, nous avons constaté que les rédacteurs n'utilisent pas des évaluations logiques mais recourent souvent à des évaluations de forme esthétique ou affective. On entend alors à des expressions telles que « ça fait lourd, ça colle pas, j'aime ça, je n'aime pas ça, mliha ».

En plus des résultats présentés ci-dessus, nous avons abouti à un autre résultat qui, à notre sens surprenant : dans la littérature de l'écrit on définit le brouillon comme la première étape dans la rédaction d'un texte. De par notre analyse, nous avons découvert que pratiquement il ya a autre brouillon qui précède le brouillon proprement dit. C'est un brouillon mental puisque généralement chaque segment de texte qu'il soit mot, groupe de mots voire phrase, est travaillé, remodelé avant d'être inscrit sur le brouillon où il pourrait subir d'autres modifications. Dès lors toutes ces modifications ou tout ce travail mental est méconnu. D'accord parce que ce travail mental ne laisse pas des traces matérielles sur une feuille mais sur le sujet écrivant. Et cela peut facilement détourner l'objectif de la rédaction: de produire un bon texte à terminer le plus vite possible le texte et s'en débarrasser, tellement cette tâche les a épuisés cognitivement et affectivement. Ceci justifie également le nombre minime sinon nul des modifications lors de la relecture du brouillon ou de la version finale. Car au terme de la rédaction, les étudiants sont déjà épuisés sinon esquintés, tellement le combat contre les mots était difficile. On assiste alors à des expressions comme « $\mathrm{A} h \mathrm{BABA} »$ à la fin de la rédaction. 


\section{Bibliographie :}

ALAMARGOT.D et CHANQUOY.L, "Apprentissage et dévellopement dans l'activité de rédaction de textes" in A.Piolat dir. Ecriture Approches en sciences cognitives, 2004, Publication de l'Université de Provence : 125-143.

BISAILLON.J, Enseigner une stratégie de révision de textes à des étudiants en langue seconde faibles à l'écrit : un moyen d'améliorer les production écrites, CIRA, 1991.

BOUCHARD.R et MONDADA.L, Les processus de la rédaction collaborative, L'Harmattan, Paris, 2005.

FAYOL.M, Des idées au texte: Psychologie cognitive de la production verbale, orale et écrite, PUF, Paris, 1997.

BRASSART.D-G, «Les processus de révision dans les modèles psycholinguistiques de la composition écrite», Recherches $\mathrm{n}^{\circ} 11$, A.F.E.F. Lilles, novembre 1989

CHANDELIER.E, Mémoire professionnel : Comment mettre en æuvre un travail de réécriture en classe de quatrième?, Académie de Lyon : centre local de Lyon, 2003-2004. DAVID.J, «La réécriture au confluent des approches linguistique, psychologique et didactique », Repères $\mathrm{n}^{\circ} 10$, 1994, pp 3-12.

DE GAULMYN.M-M, "Recherche lyonnaise sur la rédaction conversationnelle » in R.Bouchard et al., Le processus rédactionnel: écrire à plusieurs voix, L'Harmattan, Paris, 2001,pp 32-47.

DE GAULMYN. M-M, «Le corpus comme un tout » in BOUCHARD.R et MONDADA.L, les processus de la rédaction collaborative, L'Harmattan, Paris, 2005, pp17-54.

DOQUET.C, LEBLAY. C, «Temporalité de l'écriture et génétique textuelle: Vers un autre métalangage? ». Congrès Mondial de Linguistique Française, Jul 2014, Berlin, Allemagne. SHS Web of Conférences - Vol. 8 (2014) - 4e Congrès Mondial de Linguistique Française, 2014. FABRECOLS C, Réécrire à l'école et au collège, De l'analyse des brouillons à l'écriture accompagnée, Paris, ESF, 2002. Alamargot.D et .Chankoy.L in M.Fayol dir., "Les modèles de rédaction de textes » in FAYOL. M. dir., Production du langage, ed Lavoisier, 2002 : 45- 63

FAYOL M dir., Production du langage, Lavoisier, Paris, 2002.

MOUNIER.A, "Le brouillon : vers une pédagogie de la rature. Apports de la génétique textuelle en didactique de l'ecriture ». Education. 2016.

MOUNIER.A, "Le brouillon : vers une pédagogie de la rature. Apports de la génétique textuelle en didactique de l'ecriture ». Education. 2016. 
La révision dans la rédaction conversationnelle : opérations mobilisées par deux étudiants de deuxième année de licence de français.

MOULIN.A et al. "L'écriture médiatisée et distante en téléconception multisites » in R.Bouchard et al., Le processus rédactionnel : écrire à plusieurs voix, L'Harmattan, Paris, 2001, pp247-263. 